\title{
FACTORS INFLUENCING SURVIVAL FROM HAEMORRHAGIC STROKE IN AN URBAN UK CENTRE
}

\author{
C. Wharton ${ }^{1}$, A. Sadiq 1 , P. Nightingale ${ }^{2}$, S. Ispoglou ${ }^{1}$ \\ ${ }^{1}$ Sandwell and West Birmingham NHS Trust, Stroke Medicine, Birmingham, United Kingdom. \\ ${ }^{2}$ Queen Elizabeth Hospital Birmingham, Statistics, Birmingham, United Kingdom.
}

\section{Background and Aims}

Haemorrhagic stroke (HS) has a high case fatality. Established prognostic factors include clinical severity, location and volume of haematoma (1). The INTERACT2 study further showed that elevated blood glucose levels and diabetes mellitus both predict serious outcomes in patients with predominantly mild to moderate severity of acute ICH but in contrast to previous studies, did not find that diabetes predicted death after ICH (2).

Zhang et al suggested that $\mathrm{HbA1C}$ alone could serve as a better predictor of poor outcome in diabetic patients after $\mathrm{SICH}(3)$. A recent meta-analysis looking at amongst others, mortality according to age, sex and ethnic origin showed that the incidence and case fatality of $\mathrm{ICH}$ haven't changed in recent years (4).

Serving an ethnically diverse, low-income population, we retrospectively reviewed registry data to determine how certain parameters influenced 30-day survival in the cases of HS admitted to our stroke service.

\section{Methods}

We reviewed registry data from the Stroke Service at Sandwell and West Birmingham NHS Trust and looked into cases admitted between January 2010 - November 2015 which were coded as "intracerebral haemorrhage". After reviewing case records and images, $404 \mathrm{HS}$ cases were identified after excluding SAH, traumatic contusions and haemorrhagic transformation of infarcts.

We examined how age, ethnicity, gender, hypertension, diabetes, atrial fibrillation, anticoagulation, $\mathrm{HbA1C}$ and haematoma location (lobar versus deep) related to 30-day survival. Fisher's exact test was used for univariable analysis and binary logistic regression for multivariable analysis.

\section{Results}

In univariable analysis, the 30-day survival for males and females was $69 \%$ and $54 \%$ respectively $(p=0.002) .67 \%$ of patients with history of hypertension were alive at 30 days compared to $50 \%$ of patients without ( $p=0.001)$. $71 \%$ of patients with deep, and $48 \%$ with lobar haematoma were alive at 30 days $(p<0.001)$. Ethnicity and diabetes mellitus did not significantly influence this.

In multivariable analysis, age $(p<0.001)$, hypertension $(p=0.001)$ and haematoma location $(p=0.002)$ were significantly associated with 30-day survival.

In the 169 patients that $\mathrm{HbA1c}$ was available, there was no association with survival $(p=0.36)$.

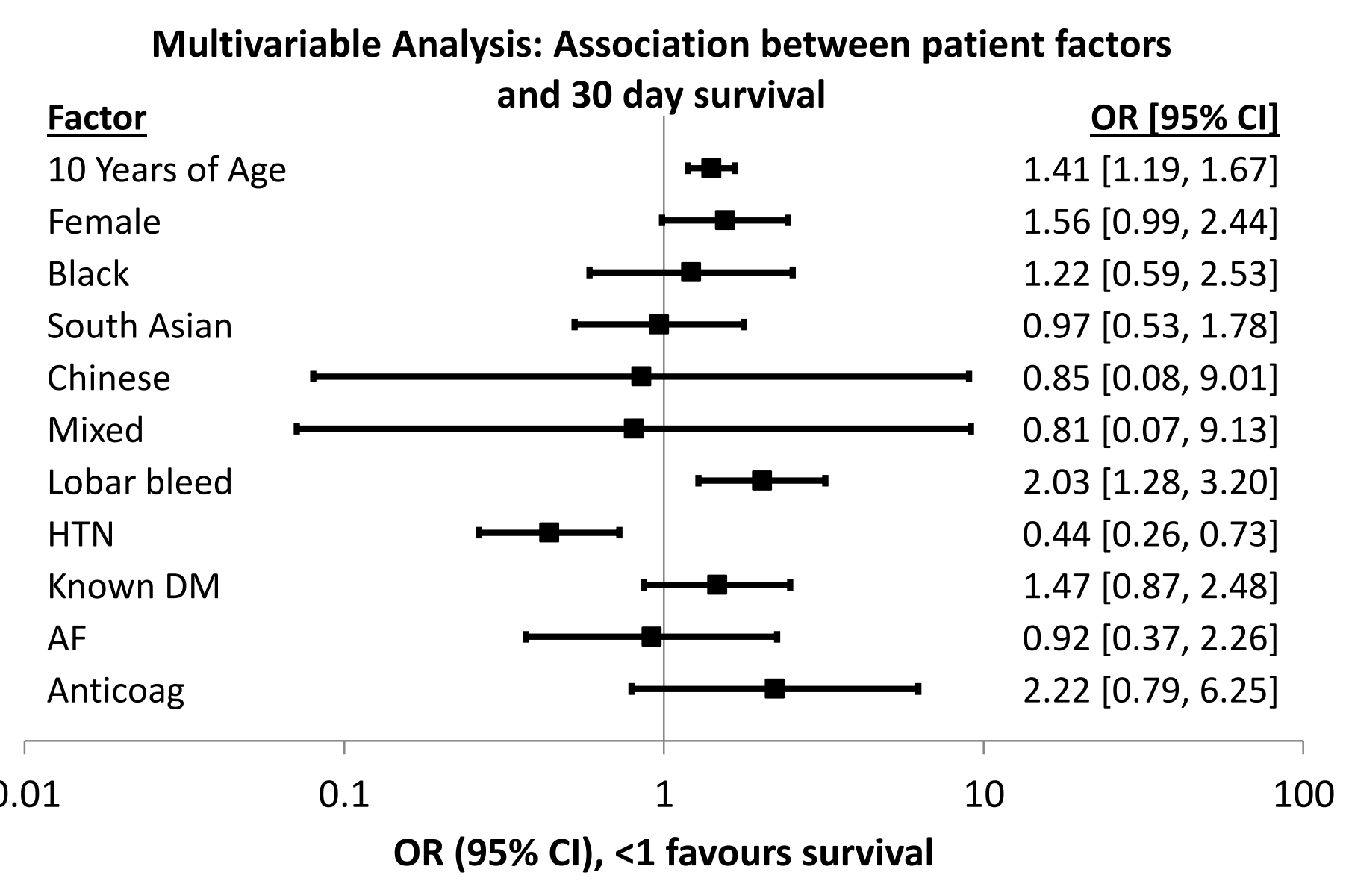

\section{Discussion and Conclusion}

In this cohort, 30-day survival was similar to what has been reported in big population studies. This was not statistically different in the ethnic groups represented. The history of hypertension favourably influenced the 30-day survival, possibly via premorbid antihypertensive treatment. Where available, HbA1c was not associated with survival. However, recognising bias due to incomplete data, retrospective analysis and no pre-specified hypothesis, we propose that a prospective study is done to determine the effect of history of hypertension and previous antihypertensive use and HbA1c on clinical outcomes from HS in the UK.

\section{References}

1. Hemphill JC III, Bonovich DC, Besmertis L, et al. The ICH score: a simple, reliable grading scale for intracerebral hemorrhage. Stroke. 2001; 32:891-897

2. Saxena et al. Prognostic Significance of Hyperglycemia in Acute Intracerebral Hemorrhage: The INTERACT2 Study. Stroke. 2016;47:682-688

3. Zhang et al. Prestroke glycaemic status is associated with the functional outcome in spontaneous intracerebral hemorrhage. Neurol Sci. 2015 36:927-934

4. Van Asch CJJ, Luitse MJA, Rinkel GJE et al. Incidence, case fatality, and functional outcome of intracerebral haemorrhage over time, according to age, sex, and ethnic origin: a systematic review and meta-analysis. Lancet Neurol 2010; 9: 167-76 